\title{
GO WITHOUT KO ON HEXAGONAL GRIDS
}

\author{
Cameron Browne ${ }^{1}$ \\ London, UK
}

\begin{abstract}
This note explains why the ko rule, which is essential for the game of Go, is not needed for hexagonal versions of the game. This known property was recently re-discovered through an evolutionary search for new games.
\end{abstract}

\section{The Ko Rule}

In order to avoid infinite cycles of play, the board game Go requires a special rule called the ko rule, which states that the player cannot make a move which would repeat the board state of the previous turn. For example, Figure 1 shows a board position (left) in which White captures a black stone by surrounding it (middle). Black cannot then immediately recapture the white piece at the point marked $\mathrm{X}$, as that would return the board state to that of the previous turn and constitute a cycle. Black must play elsewhere before making this move on a future turn.
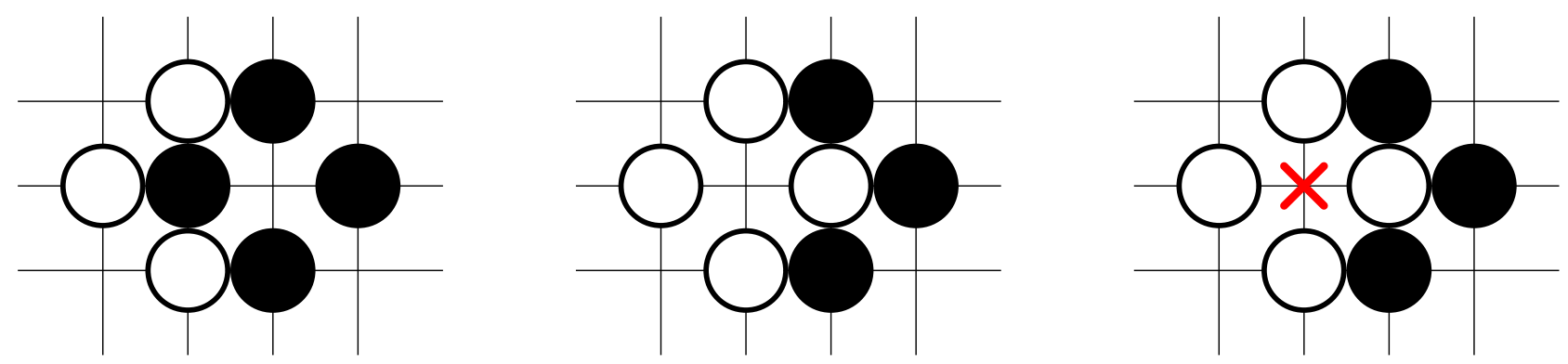

Figure 1. The ko rule; Black cannot immediately recapture at the point marked X.

The $k o$ rule has been used in Go since at least $1100 \mathrm{AD}$, as evident from game $1150 \mathrm{C} \&$ IPI2 . sg $\mathrm{f}$ between Li Baixiang and Jin Shiming, in the Games of Go on Disk (GoGoD) database (Hall, 2011). However, the general consensus among players appears to be that the ko rule has probably been used since the game's invention thousands of years ago, as some form of local cycle control is required to avoid games degenerating into infinite cycles. The $k o$ rule has since been embraced by players as a necessary part of the game, to such an extent that $k o$ battles constitute a significant field of study in their own right.

Go is still subject to the problem of triple kos, in which three separate ko occurrences conspire to give an infinite cycle of period 6, which is not handled by comparing the board state of the previous turn alone. An historical example of this is the famous "Triple Ko Game" of 1582 played at a Kyoto monastery in the presence of warlord Nobunaga; the game was abandoned, the monastery attacked, the warlord killed, and triple $k o$ has since been synonymous with bad luck. Such triple $k o$ cases require the enforcement of a stricter superko rule, which states that the player cannot make a move that repeats the board state of any previous turn. Note that this article refers to the less strict ko rule only.

\section{Hexagonal Go and Ko}

Go is undoubtedly one of the great games. It has inspired many players (and researchers), so it is only natural to attempt to map its elegant surround capture mechanism to other contexts, in an effort to find interesting variations. Changing the game's square basis to a hexagonal one is an obvious step, as the hexagonal grid has topological properties well suited to a number of other abstract board games. TriGo ${ }^{2}$ is an example of a Golike game played on a hexagonal grid. However, mapping Go to the hexagonal grid has an interesting sideeffect; the $k o$ rule is no longer needed.

\footnotetext{
${ }^{1}$ Computational Creativity Group, Imperial College London, UK. Email: camb@,doc.ic.ac.uk 

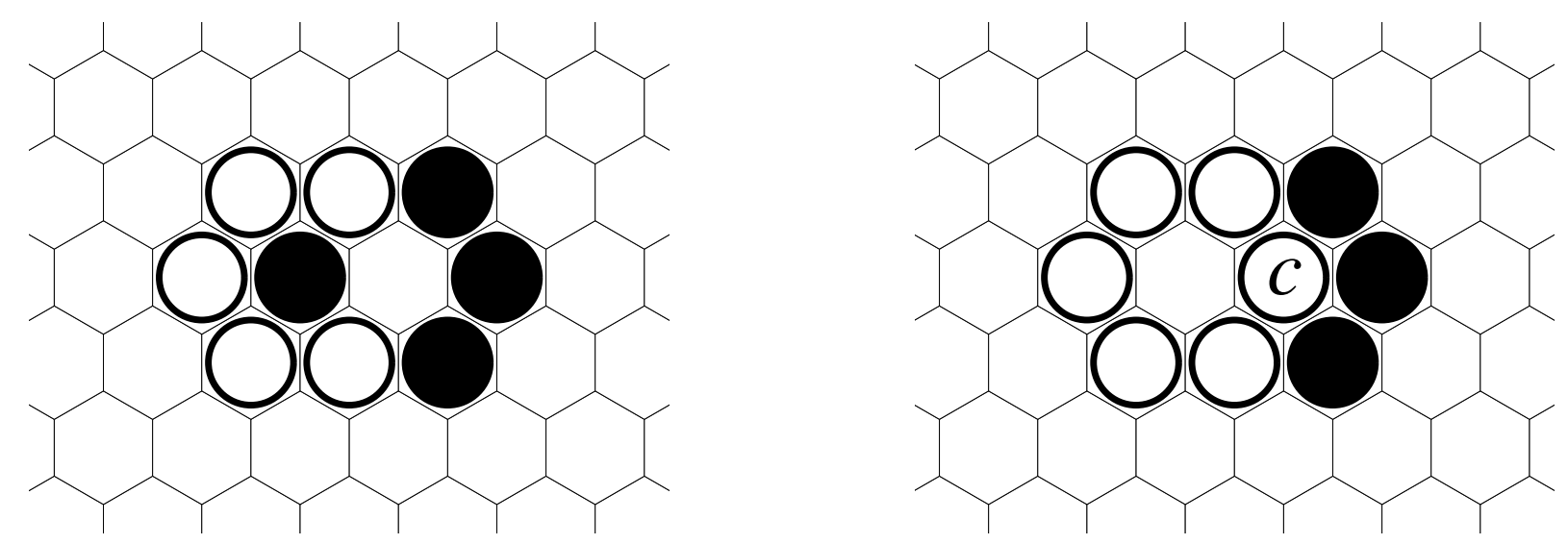

Figure 2. Immediate recapture is not possible on the hexagonal grid.

Consider the situation shown in Figure 2, which is a hexagonal analogy of the position shown in Figure 1. White can capture the black piece by surrounding it with move $c$, but now Black cannot immediately recapture the white piece just played. Local move cycles cannot occur and the ko rule does not apply here.

\section{Analysis}

The key difference between the square and hexagonal cases is that the square grid contains diagonal adjacencies and the hexagonal grid does not. This means that members of the surrounding set of capturing pieces (i.e. those pieces that remove all freedom from a group to capture it) may be diagonally adjacent on the square grid, and hence not orthogonally connected to any other piece or group, and hence vulnerable to capture themselves. On the hexagonal grid, however, there are no diagonal adjacencies; members of the surrounding set will be orthogonally connected, and the last piece played will not be subject to immediate recapture as it will become part of the surrounding group.

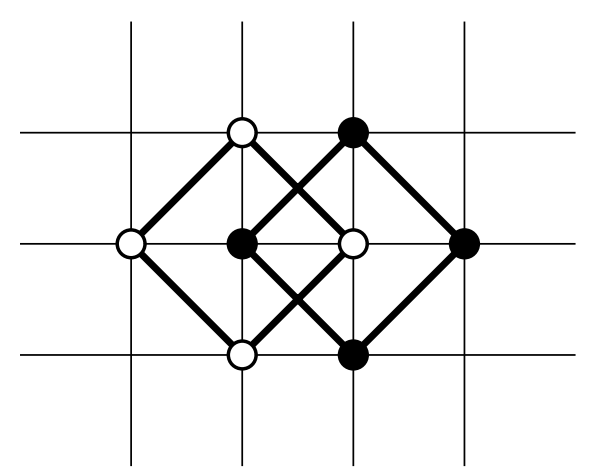

Figure 3. The connectivity graphs of the surrounding sets intersect around the capture points.

Figure 3 shows another view of the situation. The connectivity graphs of the two respective surrounding sets must intersect around the points of capture, if the surrounding sets are to recapture the capturing piece each turn. This is possible on the square grid but not on the hexagonal grid. In Go terminology, the square grid allows cross-cuts - a necessary condition for ko to occur - but the hexagonal grid does not. In mathematical terms, ko does not occur on the hexagonal grid because the neighbors of a cell are simply connected.
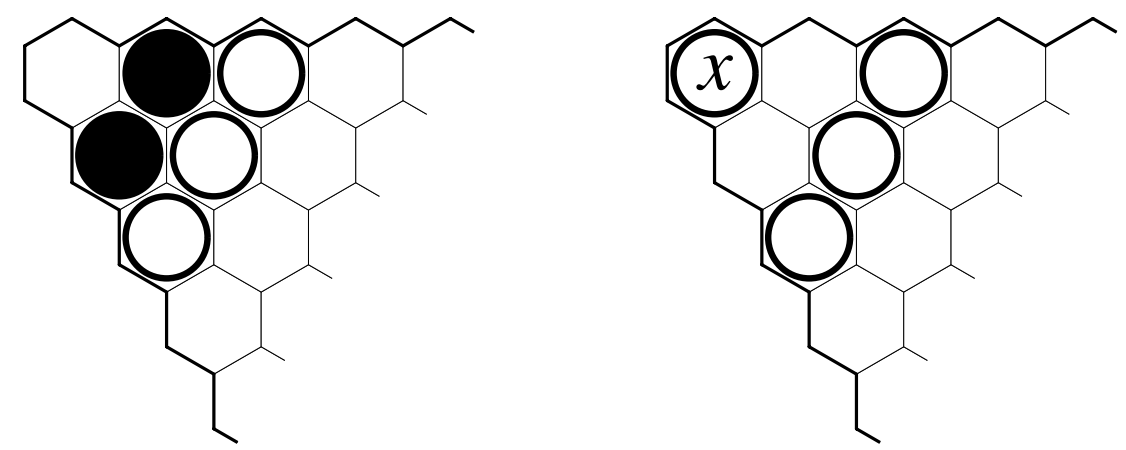

Figure 4. Immediate recapture is still not possible in an acute corner. 
Figure 4 shows a hexagonal case in which the capturing piece will not be orthogonally connected to the surrounding group, specifically when the board has one or more acute corners and the capturing piece is played in such a corner. However, there must be at least two enemy pieces separating the capturing piece from its surrounding group, hence immediate recapture is still not possible. There are other, more extreme, cases in which this condition does not hold (e.g. boards of two cells, three cells, etc.), but these are unlikely to occur in actual games.

Note that this analysis only applies to the ko rule and not the superko rule, as previously stated. Capture cycles may still be possible on the hexagonal grid, but their period will be greater than 2 , hence checking the board state of the previous turn alone will not suffice.

Game designer and publisher Néstor Romeral Andrés points out that sub-ko battles of period 3 can occur between two players of a three-player game on the hexagonal grid, if the third player conspires to allow this. For example, Figure 5 shows black and grey pieces that may perpetually capture each other while White plays elsewhere. Note that it can be beneficial to burn moves in this way rather than adding pieces to the board, according to the rules of the variant being played and whether the game has reached a cold phase.

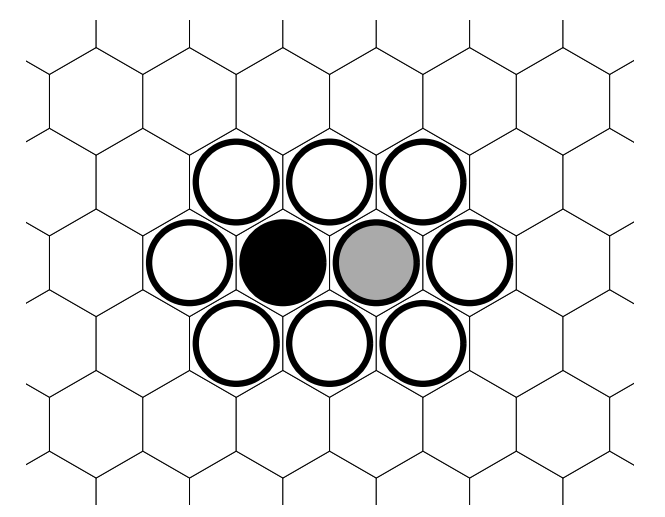

Figure 5. Ko battle between two players of a three-player game.

\section{Significance}

The non-ko property of the hexagonal grid is known among the Go-playing community. ${ }^{1}$ It has practical application in the simplification of rule sets (for example, the rules of TriGo can be simplified to remove the superfluous $k o$ rule) and in the derivation of future hexagonally-based Go variants.

The reasons for reporting this known property here - in a computer games journal - are twofold. Firstly, it has not previously been reported in the literature to our knowledge. Secondly, its recent re-discovery was inspired by the computer programme Ludi, following an evolutionary search for new and interesting games (Browne, 2011). Two of the games evolved by Ludi featured Go-like surround capture: Ndengrod, played on a hexagonal grid, and Lammothm, played on an 8-connected square grid. The ko rule was not implemented for Ludi and hence not applied in either case, yet both games survived the playability filter and scored relatively highly. In fact, Ndengrod was ranked the \#1 evolved game by both the software and by human play testers, and has since been commercially published under the name Pentalath. ${ }^{2}$ The reason for its success despite the lack of a $k o$ rule has remained something of a mystery until now.

We do not claim that Ludi re-discovered the non-ko property of the hexagonal grid, but that it implicitly captured this knowledge in its evaluations of Ndengrod and Lammothm, prompting an investigation of why the surround capture rule worked so well for these cases without $k o$. This highlights another use of the software beyond generating new games; as a creative collaborator, able to facilitate the game design process by fostering a deeper understanding of both the process and the games themselves.

\section{References}

Hall, T. M. (2011) “GoGoD Database and Encyclopaedia”, http://www.gogod.co.uk/

Browne, C. (2011) Evolutionary Game Design, Springer, Berlin.

\section{${ }_{1}^{1}$ Confirmed by Go players and game designers Bill Taylor and Stephen Tavener.}

